\title{
INTRATHALAMIC CONNECTIONS SHAPE SPINDLE ACTIVITY - A MODELLING STUDY
}

\author{
BÁlint Bús, KÁroly Antal and ZsuZsa Emri* \\ Department of Zoology, Eszterházy Károly University, Leányka u. 6, H-3300 Eger, Hungary
}

(Received: November 6, 2017; accepted: January 9, 2018)

\begin{abstract}
Spindle oscillations are generated predominantly during sleep state II, through cyclical interactions between thalamocortical and reticular neurons. Inhibition from reticular cells is critical for this activity; it enables burst firing by the de-inactivation of T-type $\mathrm{Ca}^{2+}$ channels. While the effect of different channelopathies on spindling is extensively investigated, our knowledge about the role of intrathalamic connections is limited. Therefore, we explored how the connection pattern and the density of reticular inhibitory synapses affect spindle activity in a thalamic network model. With more intrareticular connections, synchronous firing of reticular cells, and intraspindle burst frequency decreased, spindles lengthened. In models with strong intrareticular inhibition spindle activity was impaired, and a sustained 6-8 Hz oscillation was generated instead. The strength of reticular innervation onto thalamocortical cells played a key role in the generation of oscillations; it determined the amount of thalamocortical cell bursts, and consequently spindle length. Focal inputs supported bursts but affected only a few cells thus barely reinforced network activity, while diffuse contacts aided bursts only when a sufficient number of reticular cells fired synchronously. According to our study, alterations in the connection pattern influence thalamic activities and may contribute to pathological conditions, or alternatively, they serve as a compensatory mechanism.
\end{abstract}

Keywords: Burst - inhibition - nucleus reticularis thalami - spindle activity - thalamocortical cell

\section{INTRODUCTION}

The thalamus plays a primary role in generating spindle oscillations, an activity predominantly appearing during sleep stage II $[1,24,25]$. Its appropriate regulation is critical to normal brain function: the density and duration of spindles correlate with age, general intelligence, learning and problem solving ability [26]. Sleep spindles are reduced in schizophrenics [9], and share common mechanisms with absence epilepsy $[1,22]$. Genetic mutations leading to spike and wave discharges (SWD), the hallmark activity of absence epilepsy, affect sleep spindle properties as well [22].

Spindle waves are generated through cyclical interactions between thalamocortical (TC) and thalamic reticular (NRT) neurons involving both the intrinsic membrane

*Corresponding author; e-mail address: emri.zsuzsanna@uni-eszterhazy.hu 
properties of these cells and their anatomical connections [19]. Inhibition from the NRT is required to deinactivate T-type $\mathrm{Ca}^{2+}$ channels, thus preparing TC cells to produce burst firing. The strength and duration of inhibitory inputs determines the synchrony and period of the oscillation, respectively [24]. A fine balance of excitation and inhibition is required for normal thalamic function [10]. The effect of different channelopathies on this balance is extensively investigated [18], while the contribution of the connection pattern is relatively unknown. Moreover, in vitro preparations do not preserve the connection pattern completely; therefore these measurements may not reveal the changes of the network accurately. In vivo methods, like functional magnetic resonance imaging (fMRI), could expose changes in thalamocortical connectivity [8], but fMRI is unable to reveal details of intrathalamic communication. Experiments showing changes in IPSC amplitude or in the number of inhibitory synapses in epileptic animals [3] suggest that altered communication between TC and NRT cells is involved in the development of pathological thalamic functions.

Due to the limitations of experimental manipulation of the connection pattern, we explored its possible role in a network model of TC and NRT cells [6, 7]. In this model we modified the connection pattern and strength of (intra-NRT and NRT to TC) inhibitory synapses to explore their effect on the spindle oscillations.

\section{MATERIALS AND METHODS}

The network model was constructed from single compartment TC and NRT cells in the NEURON [13] modelling environment.

\section{TC and NRT cell models}

For the purpose of this study we adapted the models of NRT and TC cells used by Destexhe et al. [6, 7]. Membrane potential was governed by the following equations:

$$
C_{\mathrm{m}} \dot{V}_{\mathrm{TC}}=-g_{\mathrm{L}-\mathrm{TC}} \cdot\left(V_{\mathrm{TC}}-E_{\mathrm{L}}\right)-I_{\mathrm{T}-\mathrm{TC}}-I_{\mathrm{h}}-I_{\mathrm{KL}}-I_{\mathrm{Na}}-I_{\mathrm{K}}-I_{\mathrm{GABA}-\mathrm{A}-\mathrm{TC}}-I_{\mathrm{GABA}-\mathrm{B}}
$$

and

$$
C_{\mathrm{m}} \dot{V}_{\mathrm{NRT}}=-g_{\mathrm{L}-\mathrm{NRT}} \cdot\left(V_{\mathrm{NRT}}-E_{\mathrm{L}}\right)-I_{\mathrm{T}-\mathrm{NRT}}-I_{\mathrm{K}(\mathrm{Ca})}-I_{\mathrm{CAN}}-I_{\mathrm{Na}}-I_{\mathrm{K}}-I_{\mathrm{GABA}-\mathrm{A}-\mathrm{NRT}}-I_{\mathrm{AMPA}}
$$

where $V_{\mathrm{TC}}$ and $V_{\mathrm{NRT}}$ are the membrane potentials of TC and NRT cells, $C_{\mathrm{m}}$ the specific membrane capacity, $g_{\mathrm{L}-\mathrm{TC}}$ and $g_{\mathrm{L}-\mathrm{NRT}}$ the leakage conductances. $I_{\mathrm{T} \text {-TC }}$ and $I_{\mathrm{T}-\mathrm{NRT}}$ the T-type $\mathrm{Ca}^{2+}$ currents, $I_{\mathrm{h}}$ is the hyperpolarization-activated cation current, $I_{\mathrm{KL}}$ a leak potassium current, $I_{\mathrm{K}(\mathrm{Ca})}$ a $\mathrm{Ca}^{2+}$-activated $\mathrm{K}^{+}$current, $I_{\mathrm{CAN}}$ a $\mathrm{Ca}^{2+}$ activated cation current, $I_{\mathrm{Na}}$ and $I_{\mathrm{K}}$ the $\mathrm{Na}^{+}$and $\mathrm{K}^{+}$currents responsible for action potentials, $I_{\text {GABA-A-NRT }}$ the currents of GABAergic synapses between NRT cells, $I_{\text {GABA-A-TC }}$ and $I_{\mathrm{GABA}-\mathrm{B}}$ the GABA-A and GABA-B components of IPSPs on TC cells, and $I_{\mathrm{AMPA}}$ the 
AMPA/kainate receptors in synapses from TC to NRT cells. Conductances of synaptic currents were adjusted to match experimental behaviour: the AMPA current was set to be able to activate NRT cells following TC cell firing [11], while the input from an NRT cell did not always evoke TC burst [2].

\section{Architecture of the basic network}

We modified the network model constructed by Destexhe et al. [7] to incorporate the following anatomical findings. The intrathalamic network consists of more TC than NRT cells [16], TC cells are not interconnected [12], while some NRT cells innervate others [23]. Axonal arborization of NRT cells are heterogeneous, ranging from spatially diffuse structures with low densities of synaptic contacts to those that are focal and establish more contacts on a TC cell [5]. Reciprocal connections are rare between TC and NRT cells [20], and the divergence of inputs from NRT to TC is larger than from TC to NRT [16]. Our basic network (Fig. 1A) was constructed considering these experimental findings. Accordingly, in the "basic" model $67 \%$ of the NRT cells innervated 2 other NRT cells, thus every $3^{\text {rd }}$ NRT cells gave no intra-NRT axon. Each TC cell connected to 3 NRT cells, while each NRT cells, except the $10^{\text {th }}$, inhibited 10 TC cells. The $10^{\text {th }}$ NRT cell contacted with 16 TC cells representing the NRT cell type described by Cox et al. [5] that innervate many TC cell but forms only a few synapses on each. Between TC and NRT cells only $9.37 \%$ of the possible connections were reciprocal.

\section{Reproduction of spindle activity}

Electrophysiological recordings show 1-3 s long spindles separated by 5-20 s refractory periods, $10-14 \mathrm{~Hz}$ intraspindle burst frequency, and TC cells tend to fire only in a subset of burst cycles $[3,10]$. Blockade of the AMPA current abolishes spindle activity [25]. In the presence of bicuculline (a GABA-A antagonist) only a low-frequency oscillation can be detected and neither the blockade nor the increase of the GABA-B current changes the spindle activity drastically [15].

For each synapse type we used identical conductance values in every cell except in the $10^{\text {th }}$ NRT cells. Cox et al. [5] showed that the axonal arborization patterns of NRT cells as well as their inhibitory effects were heterogeneous, some NRT cells formed "weak" inhibitory connections with many TC cells, activating only 1-3 release sites in each. In the basic model the $10^{\text {th }}$ NRT cell represents this type: it contacts with 16 instead of $10 \mathrm{TC}$ cells and activates only a few release sites, thus the $\mathrm{G}_{\max }$ values of these synapses were set to $1 / 10$ th of the other NRT synapses.

To find models generating spindle activity with the above characteristics, we increased the maximal synaptic conductance values $\left(\mathrm{G}_{\max }\right)$ of GABA-A-NRT, AMPA synapses from $5 \mathrm{nS}$ to $50 \mathrm{nS}$ in $5 \mathrm{nS}$ steps and the $\mathrm{G}_{\max }$ values of GABA-A-TC in $1 \mathrm{nS}$ steps with all the synaptic $\mathrm{G}_{\max }$ combinations. The smaller steps were required 
to find suitable $\mathrm{G}_{\max }$ values for GABA-A-TC synapses; otherwise we could not reproduce the required level of TC cell bursting: they should not generate a burst following every NRT input, but should produce enough to sustain spindle activity. The ratio of GABA-A-TC and GABA-B $\mathrm{G}_{\max }$ was fixed to 10:1 [7]. For each GABA-A-NRT $\mathrm{G}_{\max }$, we selected a single model that reproduced spindles with the lowest AMPA and GABA-A-TC $\mathrm{G}_{\max }$ pair. In these models we studied how connections within the NRT and from NRT to TC affected spindle activity and synchronous firing within the network.

\section{Analysis of the simulations}

Bursts were defined as APs following at least $50 \mathrm{~ms}$ silent period in a cell. We defined spindles at the network level, as groups of bursts surrounded by at least $500 \mathrm{~ms}$ long silent periods. Intraspindle burst frequency was defined as the instant frequency of bursts within a spindle. Mean intraspindle burst frequency for the NRT was the average of all intraspindle burst frequencies in NRT cells. Synchrony in a nucleus was characterized by the number of cells firing in a $10 \mathrm{~ms}$ time window. Results were expressed as the mean $\pm \mathrm{SD}$.

\section{RESULTS}

\section{Basic models}

With the "basic" connection pattern (Fig. 1A) five models generating spindles with parameters similar to electrophysiological recordings (Fig. 1B) were selected. Within the tested synaptic conductance range we found models satisfying our criteria (see methods) when the $\mathrm{G}_{\max }$ values were between 0-25 nS for the GABA-A-NRT synapses, 20-25 nS for the AMPA synapses and 6-9 nS for the GABA-A-TC synapses. Models with parameters outside that range failed to reproduce spindle oscillations accurately. These models showed similar spindle length, interspindle intervals, and mean intraspindle burst frequency (Fig. 1Ca-c). Blockade of AMPA receptors hindered spindle activity (not shown). Bicuculline application resulted in an oscillation with $\sim 3.5-4 \mathrm{~Hz}$ frequency (Fig. 1Cd), while manipulating the GABA-B current varied the timings of the bursts (Fig. 1Ce). Despite the simplified rules we used for the construction of the "basic" model, cell activities varied, neither NRT nor TC cells formed groups with different levels of bursting (not shown).

\section{Inhibition and diversity within the NRT}

Altered inhibition within the NRT could reflect changes in receptor number or their subunit composition [27]. The former might reflect the reorganization of intra-NRT 


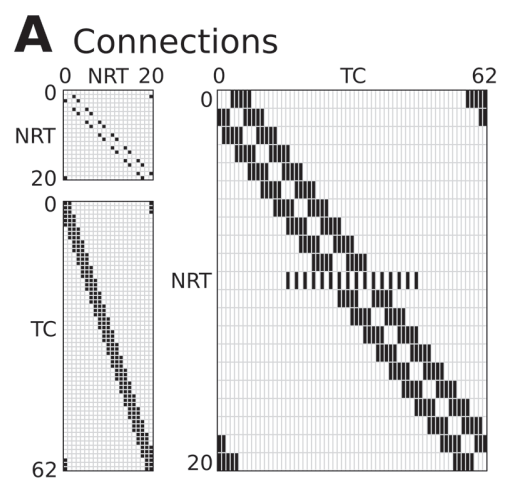

B Spindle activity

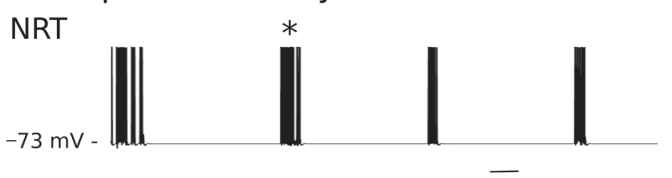

TC

$\overline{1 s}$

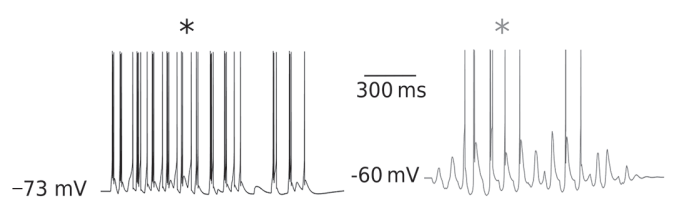

C Spindle characteristics

Ca Spindle length

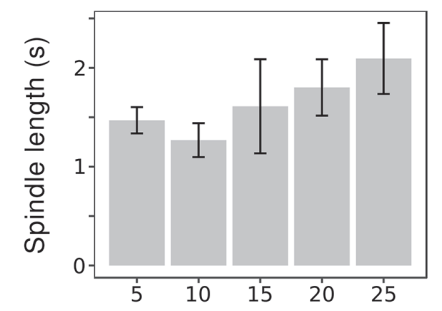

Cd No GABA-A

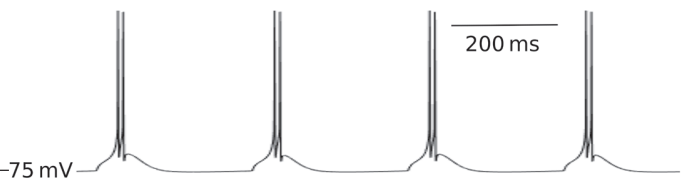

Cb Interspindle interval

Ce Altered GABA-B

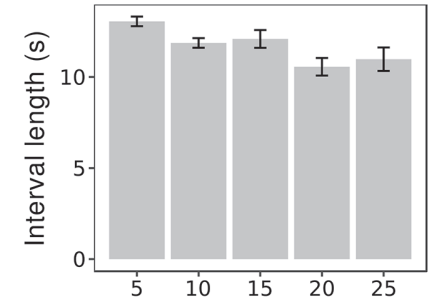

Basic

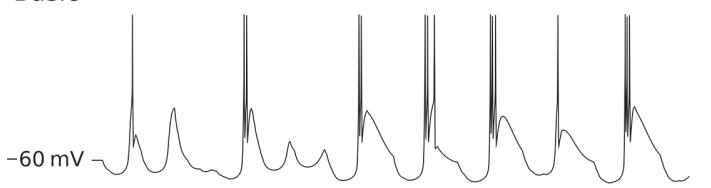

No GABA-B

Cc Burst frequency
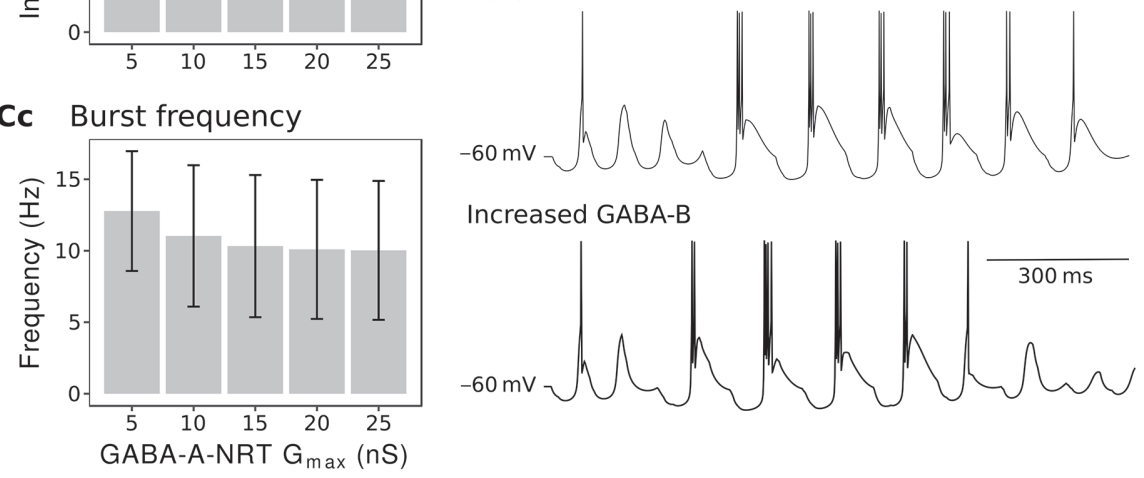

Increased GABA-B

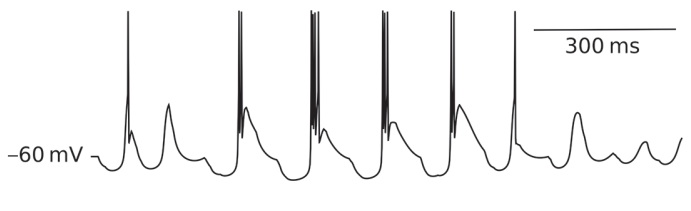


Fig. 1. Spindle characteristics in the basic models. A) Connections in the "basic" model. The model consisted of $21 \mathrm{NRT}$ and $63 \mathrm{TC}$ cells, numbered from zero. Vertical axis: source cells, horizontal: target cells. $2 / 3^{\text {rd }}$ of the NRT cells innervated two other NRT cells, and every $3^{\text {rd }}$ innervated none (left top). Each TC cell innervated 3 NRT cells (left bottom). NRT cells gave input to $10 \mathrm{TC}$ cells except the $10^{\text {th }}$ NRT cell, which innervated 16, more widely distributed TC cells (right). B) Spindle oscillations generated by the model with $15 \mathrm{nS}$ GABA-A-NRT, $20 \mathrm{nS}$ AMPA, and $8 \mathrm{nS}$ GABA-A-TC $\mathrm{G}_{\max }$ values. Stars (*) mark the spindle enlarged in the bottom. C) Graphs showing the characteristics of spindle activity in the five basic models. Models with 5-25 nS GABA-A-NRT $\mathrm{G}_{\max }$ values (horizontal axis) satisfied our criteria. Ca) Spindle lengths were between 1.0 and $2.7 \mathrm{~s}$. Cb) Interspindle intervals varied between 10.0 and 13.3 s. Cc) Mean instant burst frequencies were between 10.0 and $12.8 \mathrm{~Hz}$. Cd) No GABA-A: an oscillation with $3.5-4 \mathrm{~Hz}$ frequency was evoked when GABA-A $\mathrm{G}_{\max }=0$. Ce) Changes in the GABA-B current: simulations with basic (top), zero (middle) and increased (bottom) GABA-B G $_{\max }$. IPSPs with a GABA-B component cancelled more LTCPs, and perturbed LTCP timings more prominently than GABA-A IPSPs. Intraspindle burst frequency slightly decreased when GABA-B $\mathrm{G}_{\max }$ was doubled compared to its basic value

connections. To mimic this, we changed the number of NRT cells providing intraNRT contacts (Fig. 2A). Without intra-NRT axons the length of the spindles or the intraspindle burst frequencies were similar in all models (Fig. 2B-C, " $0 \%$ "). In the presence of intra-NRT axons, increasing GABA-A-NRT conductance and/or the number of connections increased spindle lengths and decreased mean intraspindle burst frequencies marginally (Fig. 2B-C), except in the models with "100\%" NRT connection pattern where spindle length reached $\sim 14 \mathrm{~s}$ and intraspindle burst frequencies dropped to $\sim 7 \mathrm{~Hz}$ with large GABA-A-NRT conductance (Fig. 2B-C, $25 \mathrm{nS}$, "100\%", Fig. 2Da bottom). Since varying the number of intra-NRT connections also changed the total inhibition received by NRT cells, we tested if the effects observed in " $100 \%$ " NRT connection model could be reproduced by simply scaling GABA-ANRT in the "basic" model to the same total $\mathrm{G}_{\max }$ value. "Basic" models with increased GABA-A-NRT could not reproduce the activity found in the "100\%" NRT connection models (Fig. 2Db), suggesting a role for connection patterns in shaping the activity. However, creating a small group of cells with connections similar to the " $100 \%$ " case (Fig. 2A "Group") resulted in a long second spindle with reduced burst frequency (Fig. 2Dc) resembling to the pattern found with "100\%" NRT connection models (Fig. 2Da bottom). The occurrence of synchronous firing among NRT cells was high in the models with small GABA-A-NRT $\mathrm{G}_{\max }$, and hardly changed with intra-NRT connections; bursts in the middle of a spindle recruited nearly all NRT cells and $\sim 50 \%$ of TC cells (Fig. 3A). In contrast, models with large GABA-A-NRT and "100\%" intra-NRT connections showed less synchronous firing (Fig. 3Ab). Not only the peak value changed, but also the dynamics of synchrony levels within a spindle. In the models with no intra-NRT connection the number of cells recruited to the spindle increased rapidly and then dropped abruptly (Fig. 3Ab grey), while in the models with "100\%" connection and large GABA-A-NRT, the synchronous firing especially in the case of the NRT cells changed more slowly (Fig. 3Ab black). The increase of GABA-A-NRT in the "basic" models hardly affected synchronous firings (not shown). However, in the "group" model (Fig. 3B black) we found decreased 


\section{A Intra-NRT connections}

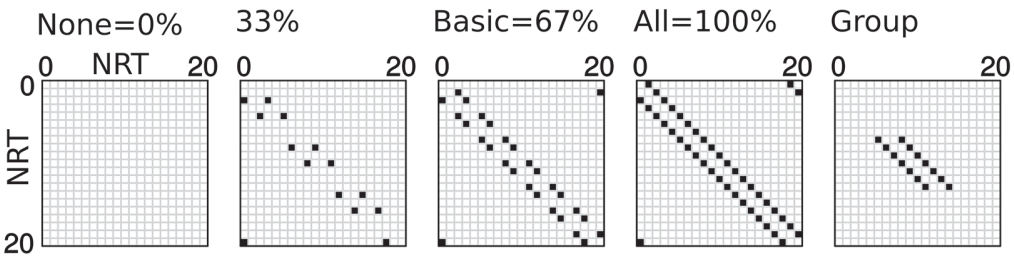

B Intraspindle frequency C Spindle length
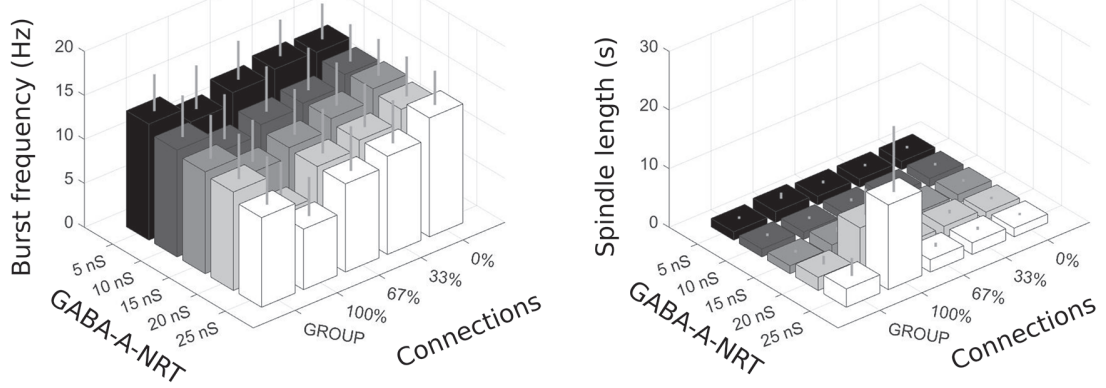

D Burst timings

Da $100 \%$ : small GABA-A-NRT

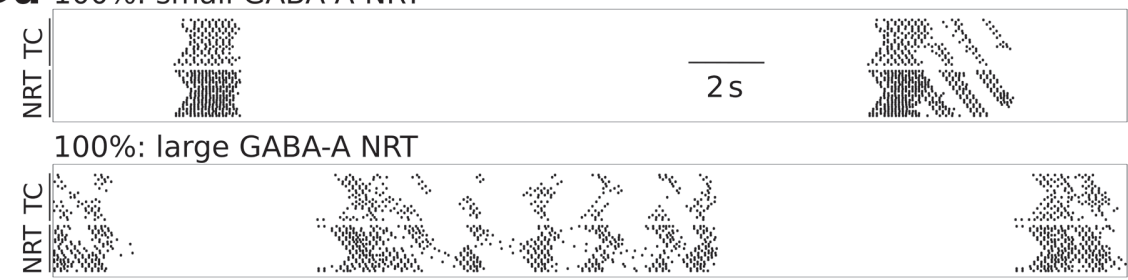

Db Basic: $1.5 x$ small GABA-A-NRT

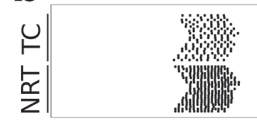

Basic: $1.5 x$ large GABA-A-NRT

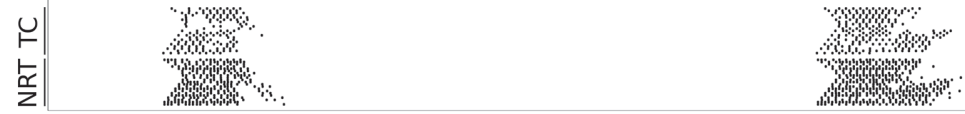

DC Group: large GABA-A-NRT

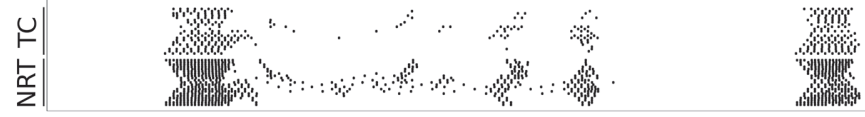


Fig. 2. Intra-NRT connections decrease burst frequency and increase spindle length. A) Intra-NRT connections: we changed the density of intra-NRT inhibition by increasing the number of cells with intraNRT axons from zero " $0 \%=$ none" to 21 "All $=100 \%$ ". Altogether five different patterns were constructed: " $0 \%=$ none", " $33 \%$ ", "Basic $=67 \%$ " and "All $=100 \%$ " refer to models within intra-NRT axons arose from 0,1 out of 3, 2 out of three, and all NRT cells, respectively. In the "Group" model the number of NRT cells providing intra-NRT axons is the same as in the "33\%" model but these connections arose from 7 consecutive NRT cells. B) The effect of intra-NRT connections on mean intraspindle burst frequency: graph shows the decrease in mean intraspindle burst frequency in the models with the five selected GABA-A-NRT values as the number of intra-NRT connections increased. The frequency reduction was the steepest in the two models with the highest GABA-A-NRT, it dropped from 9.2 to 6.7 and 6.9, when we changed GABA-A-NRT from 15 (Ns) to $20 \mathrm{Ns}$ and $25 \mathrm{nS}$, respectively. C) The effect of intra-NRT connections on spindle lengths: graph shows the variations in spindle length in the models, as the number of intra-NRT connections increased. With no intra-NRT connection, spindle length hardly varied (1.3-1.5 s); it changed more dramatically in models with high GABA-A-NRT values. In the models with " $100 \%$ " NRT connection, the spindle length increased from $1.9 \mathrm{~s}$ to $7.7 \mathrm{~s}$ when we set GABAA-NRT from $15 \mathrm{nS}$ to $20 \mathrm{nS}$. D) The effect of intra-NRT connections on burst timings: raster plots show bursting activity: with each row representing a cell, the burst timings of 21 NRT and 21 TC cells are depicted between 14 and $34 \mathrm{~s}$. Da) Burst timings in the models with "100\%" intra-NRT connection, small $(5 \mathrm{nS})$ and large $(25 \mathrm{nS})$ GABA-A-NRT $\mathrm{G}_{\max }$. With "100\%" connection bursting tended to slow down and sustain for several seconds. With large GABA-A-NRT, the first, evoked activity was that long that its end was captured in the plot. Db) Basic ("67\%") models with increased GABA-A-NRT G ${ }_{\max }(1.5 \times 5 \mathrm{nS}$ and $1.5 \times 25 \mathrm{nS}$ ). We increased GABA-A-NRT $\mathrm{G}_{\max }$ values by $50 \%$ to match the sum of the $\mathrm{G}_{\max }$ values in the "100\%" model used in Da. The enhancement induced an increase in spindle length, but it was less dramatic than the increase shown in Da. Dc) Burst timings in the 25 nS GABA-A-NRT model with "group" connection. A sustained bursting activity, similar to Da bottom, appeared in this model; however, other spindles were shorter, similar to spindles in the basic model

synchrony especially in TC cell firings compared to the "33\%" NRT connection model (Fig. 3B grey). In the "group" model synchrony was not lower throughout the spindle, during its second half more cells fired synchronously in the "group" than in the "33\%" NRT connection model (Fig. 3B).

\section{The divergence of NRT input to TC cells}

The GABA-B component of the NRT IPSPs plays a pivotal role in the deinactivation of T current, hence TC cell bursting $[15,19]$. It is significant only when NRT cells fire bursts and activate several synapses on a TC cell [15]; therefore the weight of this IPSP component changes with NRT axon ramification. To study this effect we constructed two models with altered NRT to TC connections. We kept the sum of GABAA-TC $\mathrm{G}_{\max }$ values activated by a single NRT cell constant. In the first altered connection pattern inputs from the NRT were more focused (Fig. 4A "focal"), every $2^{\text {nd }}$ NRT cell innervated only $5 \mathrm{TC}$ cells instead of 10 , while the other pattern involved diffuse NRT inputs (Fig. 4A Diffuse), each NRT cell innervated 16 instead of 10 TC cells. "Focal" inputs could evoke bursts in TC cells, but they innervated less TC cells than basic inputs, therefore overall firing, as well as the difference between NRT and TC cell activities decreased (Fig. 4Ba middle column). In models with high synchrony 

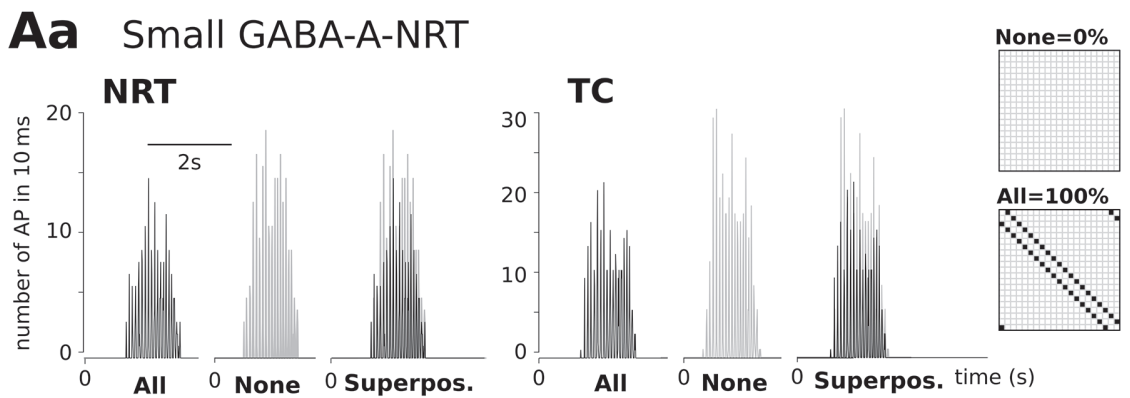

\section{Ab Large GABA-A-NRT}

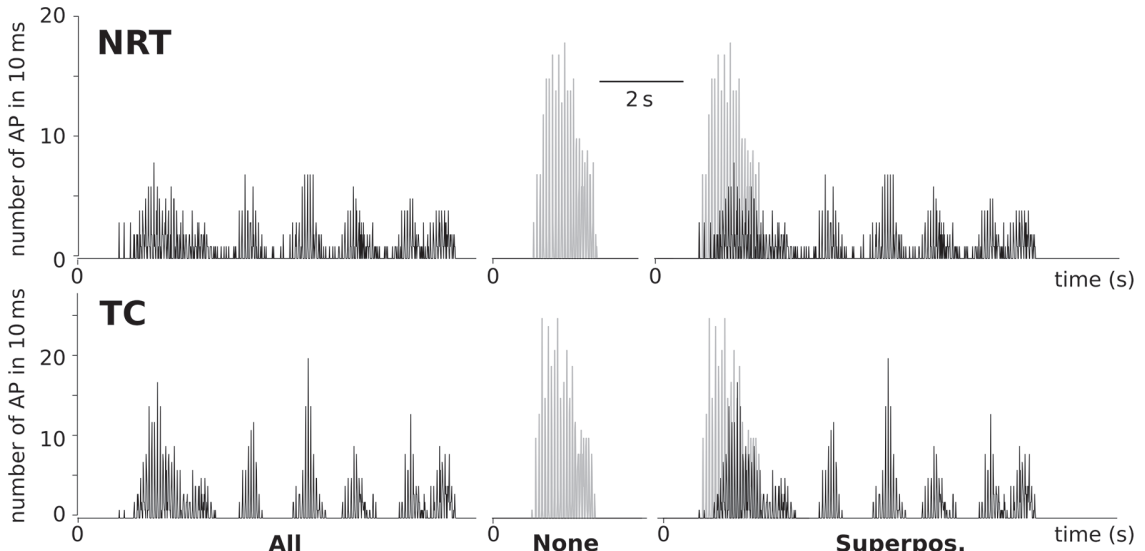

B Group, large GABA-A-NRT
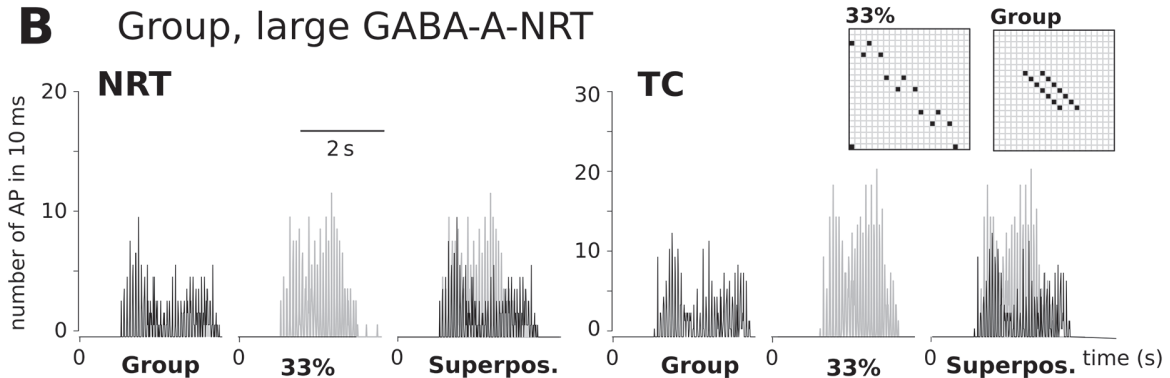

Fig. 3. The effect of intra-NRT connections on the synchrony of NRT and TC firings. Graphs show the number of NRT and TC cell firings in a $10 \mathrm{~ms}$ long time window during the $2^{\text {nd }}$ spindle. A) With intraNRT connections synchrony of cell firing decreased. Graphs show the level of synchronous firing in models with "100\%" (black) and "0\%" (grey) intra-NRT connections. Inserted diagrams show the two NRT connection patterns. Aa) In the models using small $(5 \mathrm{nS})$ GABA-A-NRT $\mathrm{G}_{\max }$ at peak nearly $100 \%$ of NRT cells and $50 \%$ of TC cells fired synchronously. Firing in the models with " $0 \%$ " and " $100 \%$ " NRT connections showed similar synchrony, the two superimposed graphs are nearly identical. Synchrony increased steeply and then dropped abruptly. Ab) In the models using large (25 nS) GABA-A-NRT synchrony with " $0 \%$ " NRT connections (grey) was similar to Aa, while with "100\%" NRT connection (black) at peak $38 \%$ of the NRT cells and $32 \%$ of the TC cells fired synchronously. In NRT cells synchrony in firing decreased less steeply, oscillation sustained for a long period (NRT, black), in TC cells peak synchrony was achieved later than in the " $0 \%$ " NRT connection model (TC black). B) The effect of intra-NRT connections on spindle length was stronger in the model where NRT cells with intra-NRT con- 
nections were grouped. Graphs from models with "group" (black) and 33\% (grey) intra-NRT connections. Inserted diagrams show the two NRT connection patterns. In the " $33 \%$ " connection at the peak $50 \%$ of NRT cells fired synchronously, and this value steeply diminished by the end of the spindle (NRT grey) while in the "group" model a comparable peak value was reached, then synchronous firing of NRT cells dropped to $20 \%$ (NRT black). The peak synchronous TC cell firing was $30 \%$ in the " $33 \%$ " NRT connection model (grey) and $17 \%$ in the "group" model. The dynamics of the change was similar except at the end of the spindle (TC black)

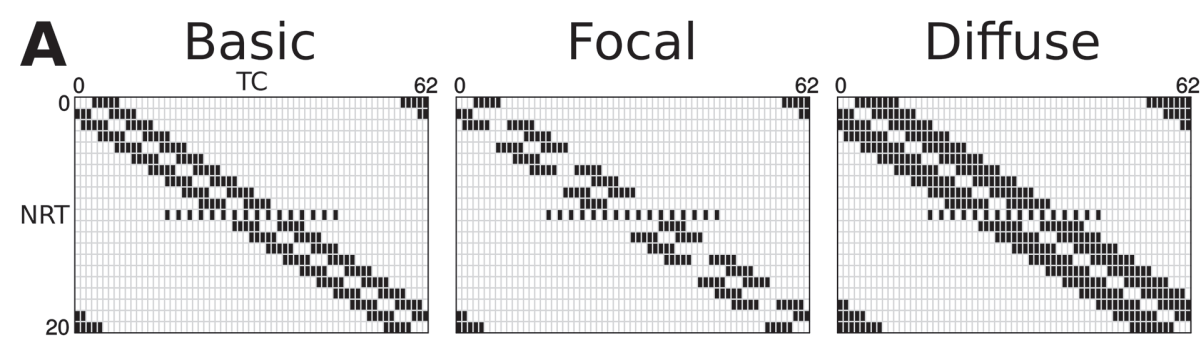

B

\section{Ba Basic NRT connection}

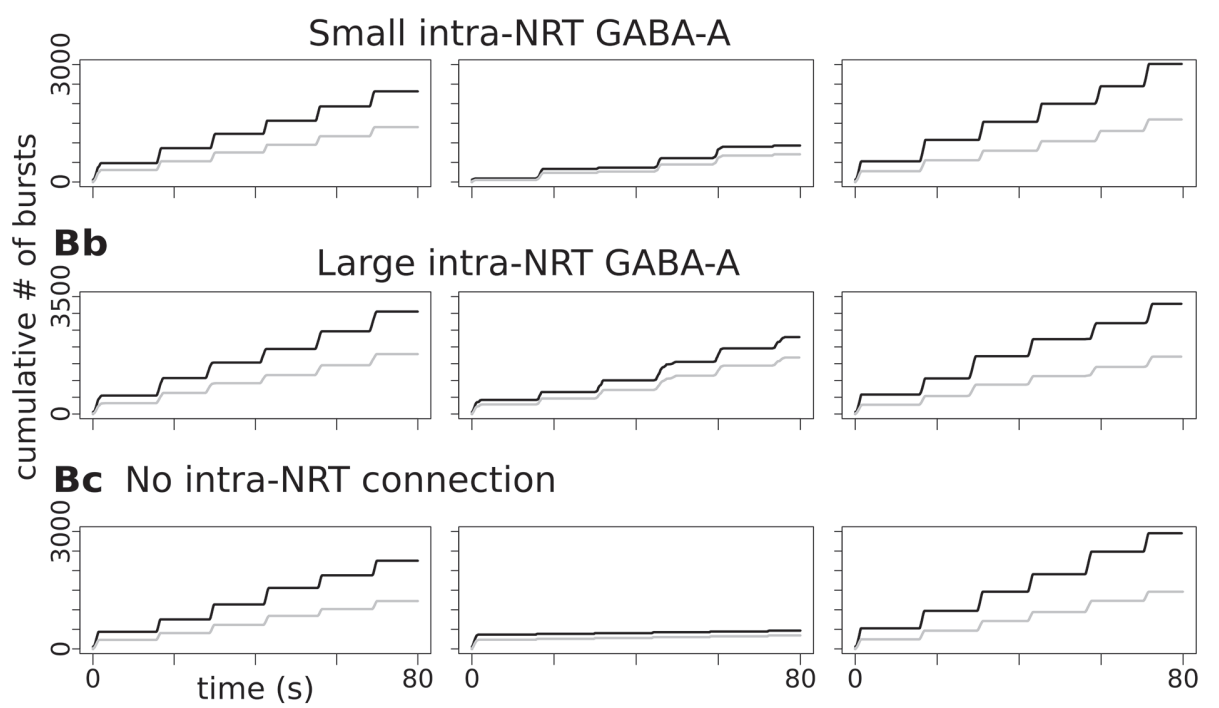

Fig. 4. Different level of convergence from NRT to TC mainly affect TC cell bursting. A) NRT to TC connection patterns. Focal connection consisted of NRT cell connecting to 5 TC cells instead of 10 . Diffuse connection pattern is composed of NRT cells innervating 16 TCs. B) Cumulative number of bursts for all NRT (black) and TC (grey) cells shown in models with NRT to TC connections illustrated above them in A. In models with basic (67\%) NRT connection either with small (5 nS; Ba) or large (25 $\mathrm{nS}$; Bb) GABA-A-NRT $\mathrm{G}_{\max }$ values the focal NRT to TC connection (middle column) showed lower activity, and more similar burst counts in NRT and TC cells than the basic model. The effect of diffuse connection (right) was the opposite, activity increased in the NRT cells therefore burst counts of the two cell types differed more in this model than in the basic model. Bc) Graphs show the activities in the model with no intra-NRT connection and high $(25 \mathrm{nS})$ GABA-A-NRT $\mathrm{G}_{\max }$ value. With focal NRT to TC connection, the oscillation stopped after a few bursts, while with diffuse NRT to TC connection the activity hardly changed 
among NRT cells (models with weak GABA-A-NRT $\mathrm{G}_{\max }$ ) after a few bursts, oscillation stopped completely (Fig. 4Bc middle); the small amount of activated TC cells could not sustain a spindle. The effect of "diffuse" inputs was the opposite, it was less effective to evoke bursts in TC cells, but reached more TC cells than the basic input, thus overall activity, and also the difference between NRT and TC cell activities increased (Fig. 4B right column). With "diffuse" connections spindles shortened slightly the weaker output to TC cells was less effective to evoke bursts than the basic input especially when only a few NRT cells fired synchronously (not shown).

\section{DISCUSSION}

We based our study on a well-established network model of NRT and TC cells [7] and kept the basic features of the circuitry, namely NRT cells inhibited each other via GABA-A synapses, and TC cells via GABA-A and GABA-B synapses while TC cells activated NRT cells via AMPA synapses and they were not connected to each other. Into this frame we incorporated additional anatomical findings to construct our "basic" connection pattern (Fig. 1A). With the tested synaptic $G_{\max }$ values several models reproduced spindle activity. While experimental findings allowed us to restrict the accepted $\mathrm{G}_{\max }$ value intervals for AMPA, GABA-A and GABA-B currents $[3,11]$, the effect of intra-NRT connections was less clearly delineated, a recent study [14] even doubted their existence, while another found that intra-NRT connections target only a subpopulation of NRT cells [4]. Nevertheless we kept the intra-NRT connections to stay consistent with the model of Destexhe [7], and to provide a source of coherence among NRT cells substituting external sources such as the basal ganglia not explicitly modelled here. We also demonstrated the feasibility of spindle activity without intra-NRT connections in the model (Fig. 2).

When we changed the number of intra-NRT connections, we found that increased intra-NRT inhibition lengthened spindles, decreased spindle frequency and the level of synchrony. In this model $\mathrm{Ca}^{2+}$-dependent augmentation of $\mathrm{I}_{\mathrm{h}}$ causes depolarization and ceases oscillation [7]. Intra-NRT inhibition, by decreasing the synchrony and the frequency of NRT cell bursts and consequently TC cell bursts, lessened $\mathrm{Ca}^{2+}$ dependent augmentation of $I_{\mathrm{h}}$, thus sustained oscillations longer. Several studies showed the opposite: increased intra-NRT inhibition was antiepileptic and decreased inhibition proepileptic [21,23], suggesting a role for other mechanisms not included in this model. It should also be noted that in these experiments all NRT-GABA-A receptors were modified uniformly, while the rearrangement of intra-NRT changed the strength of the connections non-uniformly. Alternatively, the presence of another NRT cell type, described by Clemente-Perez et al. [4], could explain the discrepancy between our simulations and experimental results. According to their study the NRT cells that receive the majority of the intra-NRT input are responsible for only the minority of NRT or TC cell bursting in sensory thalamic nuclei, thus hardly affect oscillatory activities, unlike the NRT cells in the model. However, the increased susceptibility of mice lacking T type $\mathrm{Ca}^{2+}$ channels in NRT cells to drug-induced SWD 
could be explained by the mechanism we found in the model: in these mice increased tonic firing of NRT cells is able to support long-lasting oscillations with 4-12 Hz frequencies, when GABA-B current is increased [17].

Next, we changed the number of TC cells innervated by a single NRT cell without affecting the sum of the outgoing synaptic $G_{\max }$ values. In the "focal" model this yielded large GABA-AB IPSPs, but only in a few TC cells while in the "diffuse" model a single NRT cell elicited small IPSPs in several TC cells. The net effect depended on the synchrony of NRT inputs, and in turn, the number of TC cells exhibiting large GABA-B IPSPs. This finding offers an explanation to apparently contradictory experimental results: increased GABA-B IPSPs promote slow epileptic discharges, when they affect many TC cells [15], but desynchronize the network when they are evoked in only a few TC cells [24].

In summary, we showed that changes in the connection pattern affect the activity of the thalamic network and their effect is shaped by the balance among synaptic currents. While the modulation of the connections between NRT and TC cells altered spindle oscillations similarly to experimental findings, the rearrangement of intraNRT connection did not mimic activity changes found in several models of absence epilepsy.

\section{ACKNOWLEDGEMENT}

The research was supported by the grant EFOP-3.6.1-16-2016-00001 ("Complex improvement of research capacities and services at Eszterházy Károly University").

\section{REFERENCES}

1. Avoli, M. (2012) A brief history on the oscillating roles of thalamus and cortex in absence seizures. Epilepsia 53, 779-789.

2. Barthó, P., Slézia, A., Mátyás, F., Faradzs-Zade, L., Ulbert, I., Harris, K. D., Acsády, L. (2014) Ongoing network state controls the length of sleep spindles via inhibitory activity. Neuron 82, $1367-1379$

3. Cavdar, S., Hacıoğlu, H., Doğukan, S. Y., Onat, F. (2012) Do the quantitative relationships of synaptic junctions and terminals in the thalamus of genetic absence epilepsy rats from Strasbourg (GAERS) differ from those in normal control Wistar rats? Neurol. Sci. 33, 251-259.

4. Clemente-Perez, A., Makinson, S. R., Higashikubo, B., Brovarney, S., Cho, F. S., Urry, A., Holden, S. S., Wimer, M., Dávid, C., Fenno, L.E., Acsády, L., Deisseroth, K., Paz, J. T. (2017) Distinct thalamic reticular cell types differentially modulate normal and pathological cortical rhythms. Cell Rep. 19, 2130-2142.

5. Cox, C. L., Huguenard, J. R., Prince, D. A. (1997) Nucleus reticularis neurons mediate diverse inhibitory effects in thalamus. Proc. Natl. Acad. Sci. U. S. A. 94, 8854-8859.

6. Destexhe, A., Contreras, D., Sejnowski, T. J., Steriade, M. (1994) A model of spindle rhythmicity in the isolated thalamic reticular nucleus. J. Neurophysiol. 72, 803-818.

7. Destexhe, A., Bal, T., McCormick, D. A., Sejnowski, T. J. (1996) Ionic mechanisms underlying synchronized oscillations and propagating waves in a model of ferret thalamic slices. J. Neurophysiol. 76, 2049-2070

8. Fama, R., Sullivan, E. V. (2015) Thalamic structures and associated cognitive functions: Relations with age and aging. Neurosci. Biobehav. Rev. 54, 29-37. 
9. Ferrarelli, F., Tononi, G. (2011) The thalamic reticular nucleus and schizophrenia. Schizophr. Bull. 37, 306-315.

10. Fogerson, P. M., Huguenard, J. R. (2016) Tapping the brakes: cellular and synaptic mechanisms that regulate thalamic oscillations. Neuron 92, 687-704.

11. Gentet, L. J., Ulrich, D. (2003) Strong, reliable and precise synaptic connections between thalamic relay cells and neurones of the nucleus reticularis in juvenile rats. J. Physiol. 546, 801-811.

12. Grant, E., Hoerder-Suabedissen, A., Molnár, Z. (2012) Development of the corticothalamic projections. Front Neurosci. 6, 53.

13. Hines, M., Carnevale, T. (2015) NEURON simulation environment in: Jaeger, D., Jung, R. (eds) Encyclopedia of Computational Neuroscience. Springer Science+Business Media, New York, pp. 2012-2017.

14. Hou, G., Smith, A. G., Zhang, Z. W. (2016) Lack of intrinsic GABAergic connections in the thalamic reticular nucleus of the mouse. J. Neurosci. 36, 7246-7252.

15. von Krosigk, M., Bal, T., McCormick, D. A. (1993) Cellular mechanisms of a synchronized oscillation in the thalamus. Science 261, 361-364.

16. Lam, Y. W., Sherman, S. M. (2005) Mapping by laser photostimulation of connections between the thalamic reticular and ventral posterior lateral nuclei in the rat. J. Neurophysiol. 94, 2472-2483.

17. Lee, S. E., Lee, J., Latchoumane, C., Lee, B., Oh, S. J., Saud, Z. A., Park, C., Sun, N., Cheong, E., Chen, C. C., Choi, E. J., Lee, C. J., Shin, H. S. (2014) Rebound burst firing in the reticular thalamus is not essential for pharmacological absence seizures in mice. Proc. Natl Acad. Sci. U. S. A. 111, $11828-121833$.

18. Maheshwari, A., Noebels, J. L. (2014) Monogenic models of absence epilepsy: windows into the complex balance between inhibition and excitation in thalamocortical microcircuits. Prog. Brain Res. 213, 223-252.

19. McCormick, D. A., Bal, T. (1997) Sleep and arousal: thalamocortical mechanisms. Annu. Rev. Neurosci. 20, 185-215.

20. Pinault, D. (2004) The thalamic reticular nucleus: structure, function and concept. Brain Res. Revs, $46,1-31$

21. Schofield, C. M., Kleiman-Weiner, M., Rudolph, U., Huguenard, J. R. (2009) A gain in $\mathrm{GABA}_{\mathrm{A}}$ receptor synaptic strength in thalamus reduces oscillatory activity and absence seizures. Proc. Natl Acad. Sci. U. S. A. 106, 7630-7635.

22. Sitnikova, E., Hramov, A. E., Grubov, V., Koronovsky, A. A. (2014) Time-frequency characteristics and dynamics of sleep spindles in WAG/Rij rats with absence epilepsy. Brain Res. 1543, 290-299.

23. Sohal, V. S., Huguenard, J. R. (2003) Inhibitory interconnections control burst pattern and emergent network synchrony in reticular thalamus. J. Neurosci. 23, 8978-8988.

24. Sohal, V. S., Pangratz-Fuehrer, S., Rudolph, U., Huguenard, J. R. (2006) Intrinsic and synaptic dynamics interact to generate emergent patterns of rhythmic bursting in thalamocortical neurons. J. Neurosci. 26, 4247-4255.

25. Tancredi, V., Biagini, G., D’Antuono, M., Louvel, J., Pumain, R., Avoli, M. (2000) Spindle-like thalamocortical synchronization in a rat brain slice preparation. J. Neurophysiol. 84, 1093-1097.

26. Ujma, P. P., Konrad, B. N., Genzel, L., Bleifuss, A., Simor, P., Pótári, A., Körmendi, J., Gombos, F., Steiger, A., Bódizs, R., Dresler, M. (2014) Sleep spindles and intelligence: evidence for a sexual dimorphism. J. Neurosci. 34, 16358-16368.

27. Zhou, C., Ding, L., Deel, M. E., Ferrick, E. A., Emeson, R. B., Gallagher, M. J. (2015) Altered intrathalamic GABAA neurotransmission in a mouse model of a human genetic absence epilepsy syndrome. Neurobiol. Dis. 73, 407-417. 